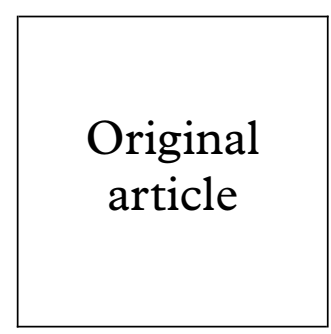

\title{
HIV infection among family planning clinic attenders in Glasgow: why prevalence has remained low in this general population group
}

\author{
D Goldberg, S Carr, S Cameron, G Codere
}

\begin{abstract}
Objective: During 1991-2, unlinked anonymous testing of dried blood spots from neonatal metabolic screening cards showed the prevalence of HIV among childbearing women from Glasgow to be extremely low at $0.01 \%$. A study was conducted to determine if non-pregnant sexually active women who engaged in unprotected sexual intercourse were more likely to be infected than those who were pregnant.

Methods: Unlinked anonymous HIV testing of urine specimens submitted by attenders of the family planning clinic in Glasgow for pregnancy testing.

Results: Of 11990 urine specimens tested, 7664 were from women with a negative pregnancy test and two of these were HIV positive $(0.026 \%)$; none of the remainder from those with a positive pregnancy test had HIV antibodies.

Conclusion: No hidden epidemic was unearthed among a population which had engaged in unprotected sexual intercourse and was not pregnant. Other data from Glasgow strongly suggest that the control of HIV transmission among the city's population of current injectors (HIV prevalence, $1 \%$ of 8500 ) has prevented the spread of infection into its wider heterosexual population. It is essential that preventive measures which have been responsible for this public health success should be maintained.
\end{abstract}

(Sex Transm Inf 1998;74:50-53)

Keywords: HIV prevalence; Glasgow; family planning; urine testing

\section{Introduction}

During the mid to late 1980s investigators from a wide range of disciplines expended considerable energy in designing surveys and surveillance programmes to measure the prevalence of HIV infection in high and low risk population groups. ${ }^{1}$ The resulting data would help personnel working in the HIV/AIDS field to target, evaluate, and implement prevention initiatives, to make informed decisions about the distribution of resources for the health and social care of infected individuals, and to promote research and education. By early 1990, sex workers, injecting drug users, homosexual/bisexual males, heterosexual attenders of genitourinary clinics, and pregnant women were undergoing anonymous HIV testing.

Determining the prevalence of HIV among pregnant women was particularly important because it would help gauge the extent to which infection had spread into the wider heterosexual population. This group has particular characteristics which make it appropriate for such an epidemiological study. These include: (i) they have all engaged in unprotected vaginal intercourse; (ii) since the heterosexual transmission of HIV is more efficient from male to female than vice versa, the earliest signs of heterosexual spread would be more likely to be detected in this population; and (iii) the sample is generally large, geographically widespread, and prone to only minimal demographic variability over time. For the entire childbearing population in Scotland, a programme of unlinked anonymous HIV testing of dried blood spots from neonates was implemented in January $1990 .{ }^{2}$ Since HIV antibodies cross the placenta from the mother to the fetus, a seropositive neonate indicates maternal infection.

However, it was recognised that the prevalence of HIV could be different in young sexually active women engaging in unprotected or inadequately protected sexual intercourse who were not pregnant. These women would include (i) those who were infertile as a consequence of, for example, sexually transmitted chlamydial infection, ${ }^{3}$ or (ii) individuals who did not wish to become pregnant but were having a pregnancy test because of a lapse in hormonal contraceptive administration; some of these women might engage in particularly high levels of unprotected sexual intercourse and thus be at higher risk of acquiring HIV. Unlinked anonymous testing, which is the most cost efficient means of monitoring HIV prevalence in a general population group, can only be performed on residual fluids from specimens which have been taken for another clinical purpose. Since it had already been shown that certain assays could provide accurate HIV test results when applied to urine specimens, ${ }^{4}$ women submitting a urine sample for pregnancy testing were considered an appropriate population to study; of particular interest were the majority whose pregnancy tests were negative.

A pilot study was conducted in a large family planning clinic in Glasgow, Scotland, where the prevalence of HIV among childbearing women during 1991-2 was known to be 
$0.01 \%{ }^{2}$ to determine (i) if HIV prevalence in a non-childbearing population was different from that among women giving birth, and (ii) if it was feasible to conduct such surveillance in a busy clinical setting.

\section{Methods}

SETTING

Glasgow's family planning and sexual health service is the largest of its type in the United Kingdom and its city centre clinic has approximately 1000 attendees per week. This clinic serves the city of Glasgow, particularly its central areas, and $91.7 \%$ of women attending for a pregnancy test during the study period had a Glasgow postal code of residence.

\section{SUBJECTS AND ELIGIBILITY}

All women who attended the clinic for a pregnancy test during January 1992 to December 1994 were eligible for entry into the study. Literature explaining that their urine specimens might be tested anonymously for HIV was accessible to all; any woman could object to the HIV test being performed.

\section{REPRESENTATIVENESS}

Clinic attenders who have a negative pregnancy test result are not a representative sample of sexually active non-pregnant women in Glasgow. However, this population was considered the most representative of all those available for study when taking into consideration the need for clinical specimens on which to perform unlinked anonymous HIV testing. The family planning clinic provides greater anonymity and confidentiality than the general practice and, accordingly, attracts certain groups of women at higher risk of acquiring HIV such as the partners of injecting drug users and men from Africa. ${ }^{5}$ While some selection bias does exist, this cannot be said for participation bias which is negligible as a consequence of the unlinked anonymous testing approach.

\section{CLINIC PROCEDURE}

Following the pregnancy test which is performed routinely on a urine specimen, the result was transcribed onto each of a pair of adhesive labels bearing an identical study number. One label was applied to a study urine container and the other to the clinical audit sheet which is routinely attached to each patient's case record. Residual urine left after pregnancy testing was decanted into the study container which was then stored temporarily at $4^{\circ} \mathrm{C}$.

The following patient details were transcribed from the clinical audit sheet on to the study computer; study number, date of specimen, patient's initials, date of birth, first part of postal code of residence, and pregnancy test result. A duplicate search was performed (using initials and date of birth as identifiers) to ensure that only the first urine specimen submitted by a woman within a rolling 12 month period would be tested anonymously for HIV. The study numbers corresponding to specimens eligible for testing were generated monthly; these were listed adjacent to adhesive labels bearing a code which indicated the woman's non-identifying details of age band, calendar quarter of specimen, pregnancy test result, and first part of postal code. Using the study number to identify the appropriate urine specimen, the coded label was then applied to its corresponding container. The study number was then removed from the container, thus anonymising the sample. Batches of samples were then despatched to the west of Scotland HIV reference laboratory at Ruchill Hospital.

\section{LABORATORY PROCEDURE}

All specimens were screened for HIV-1 antibodies by an IgG antibody capture particle adherence test (GACPAT in house). The sensitivity of the test was controlled by the inclusion of a weak HIV positive urine specimen. Reactives were then tested by an IgG antibody capture enzyme linked immunosorbent assay (Gacelisa, Murex, Biotech). A western blot (HIV-1 miniblot Organon Teknika) assay was used to confirm these reactives; the criteria of positivity were the presence of p24 gp 160/120 and another band.

\section{Results}

From January 1992 to December 1994, 11990 urine specimens were tested for HIV antibodies; 7664 of these were from women with a negative pregnancy test and two were HIV positive $(0.026 \%)$. None of the 4326 specimens from those with a positive pregnancy test had antibodies for HIV. Both HIV positive specimens originated from women aged 25 to 29 whose pregnancy tests were negative and who were resident in a local postcode area. Only one spontaneous objection was noted throughout the study period.

\section{Discussion}

The findings of this study indicate that in Glasgow during 1992-4 the prevalence of HIV infection among non-pregnant but sexually active young women attending the city's main family planning clinic for a pregnancy test was extremely low and similar to that observed in the pregnant group. While it is recognised that a women will often belong to both groups but at different times, it is reassuring that no hidden epidemic was unearthed among a population which had engaged in unprotected sexual intercourse and was not pregnant. The study also demonstrated that such a surveillance system can be implemented and managed successfully in a busy clinic setting without disrupting its routine.

An explanation of the low HIV prevalence found in women attending the family planning clinic in Glasgow can be achieved by placing this observation in the context of other available data concerning the spread of HIV in this city. Greater Glasgow (population 960 000) has an estimated 8500 current injecting drug users ${ }^{6}$ and, accordingly, has one of the highest rates of injecting in the world. While HIV infection entered the Glasgow injector population in the mid 1980s, the city did not experience the epidemic spread which occurred simultaneously among injecting 
populations in Edinburgh ${ }^{78}$ and Dundee. ${ }^{9}$ During the early 1990s, numerous studies of Glasgow injecting drug users who were tested in drug treatment centres, ${ }_{110}^{10}$ street settings, ${ }^{10}$ needle/syringe exchanges, ${ }^{110}$ prisons, ${ }^{11-14}$ genitourinary clinics, ${ }^{1}$ a healthcare centre for sex workers, ${ }^{15-17}$ and National Health Service HIV testing centres ${ }^{18}{ }^{19}$ detected prevalence rates which averaged around $1 \%$ and never exceeded $3.5 \%$. Only in Glenochil Prison, central Scotland, where an outbreak of HIV in early 1993 involved a minimum of 13 injectors from Glasgow has there been any evidence of appreciable transmission in this group since the 1980s. ${ }^{1120}$

There is considerable evidence to indicate that the implementation of a needle/syringe exchange programme in Glasgow during 1988 has resulted in a decrease in the sharing of injecting equipment and thus the control of infection among injectors. ${ }^{21-23}$ For example, in Glasgow, during 1990-4, the great majority of injectors never, or only occasionally, injected with used needles and those attending needle/ syringe exchanges were far less likely to share equipment than those who did not. ${ }^{24}$

Since the prevalence of HIV among injectors is only $1 \%$, the opportunity for the spread of infection from injectors to their non-injecting heterosexual partners is low. As at December 1994, only eight cases among sexual contacts of injectors in Glasgow had been detected. Another route of entry for HIV into the heterosexual population is through an infected bisexual male having unprotected sexual intercourse with a female partner. While various studies have shown a 5\% HIV prevalence among homosexual/bisexual males in Glasgow during the $1990 \mathrm{~s},{ }^{18}$ only two cases among female contacts of bisexuals have been reported. If there is minimal heterosexual transmission of HIV emanating from injectors and bisexual males in Glasgow, the only other potential source is from abroad. Between January 1991 and December 1995, 21500 noninjecting heterosexual men and women who attended genitourinary clinics in Glasgow with a sexually transmitted disease problem were tested anonymously for $\mathrm{HIV}^{25}$ Prevalence among 13000 and 7500 females was $0.23 \%$ and $0.09 \%$ respectively. However, when prevalence among people declaring any nationality or location associations with Africa were compared with rates among those with United Kingdom connections only, major differences were seen. For the United Kingdom only group, the prevalence rates among 9700 males and 6300 females were $0.09 \%$ and $0.02 \%$ respectively while those for the corresponding groups with African associations were $3.06 \%$ $(11 / 359)$ and $2.58 \%(4 / 155) .{ }^{25}$ Interestingly, the HIV prevalence among females with United Kingdom only connections $(0.02 \%)$ is identical to that seen among the family planning clinic attenders.

Unlinked anonymous HIV testing of dried blood spots on neonatal metabolic screening cards from approximately 12000 babies from Greater Glasgow each year has shown prevalence rates in childbearing women of between
$0 \%$ and $0.02 \%$ during $1992-4 .{ }^{18}$ It is also worth noting that the prevalence of HIV among blood donations from female heterosexuals in Glasgow during 1990-3 was 0\%.

It is now possible to appreciate why the HIV prevalence among family planning clinic attenders has remained so low. In Glasgow, it is uncommon to find HIV in the heterosexual population and where infection is detected it is invariably in someone who injects drugs or who has had sexual intercourse with an African partner. The findings of the family planning clinic study, particularly when placed alongside other data, provides an insight into the dynamics of HIV transmission in a typical urban area in the United Kingdom. Outside London, Edinburgh, and Dundee, most cities in the United Kingdom appear to have similar population profiles of risk - that is, one in 20 for homosexual/bisexual males, one in 100 for drug injectors, and one in 5000-10000 for women of childbearing age. ${ }^{26}$

The control of HIV transmission among injectors in Glasgow has prevented spread into the wider heterosexual population; infections have occurred in sexual contacts of injectors but these are rare. Heterosexuals at most risk are those who travel between the United Kingdom and countries with a high HIV prevalence and, accordingly, HIV prevention initiatives should be targeted at this group. Nevertheless, it is critical that initiatives which have successfully controlled the spread of HIV among injectors and thus from them to their noninjecting partners should be maintained.

We would like to thank Linda MacDonald, and Karen Wilson for carrying out the laboratory work at the Regional Virus Larang Laboratory, Ruchill Hospital, Glasgow; Muriel Holroyd, Nan Leckie, and Karen Shanlin for work conducted at the family planning clinic, Claremont Terrace, Glasgow; and William the Scottish Centre for Infection and Environmental Health.

$1 \mathrm{HIV}$ and AIDS Surveillance in Scotland. Review of the Epidemic to December 1994. ANSWER (AIDS News Supplement to the Weekly Report) December 1995:special

Tappin DM, Girdwood RWA, Follett EAC, et al. Prevalence of maternal HIV infection in Scotland, based on unlinked anonymous testing of newborn babies. Lancet 1991;337 1565-7.

3 Paavonen J. Is screening for Chlamydia trachomatis infection cost effective? Genitourin Med 1997;73:103-4.

4 Connell JA, Parry JV, Moramer PP, et al. Preliminary report: accurate assays for anti-HIV in urine. Lancet 1990;335 1366-9.

5 Reid M, Murray K, Graham N, Holroyd M, Bigrigg A Information, advice and counselling: a study of non-clinical aspects of the family planning services. $\mathrm{Br} \mathcal{F}$ Fam Plan 1996;22:112-6

6 Frischer M, Leyland A, Cormack R, et al. Estimating population prevalence of injected drug use and HIV infection among injecting drug users in Glasgow. Am f Epidemiol 1993;138: $170-81$.

7 Robertson JR, Bucknall ABV, Welsby PD, et al. Epidemic of AIDS related virus (HTLV III/LAV) infection among intravenous drug users. BMF 1986;292:527-9.

8 Peutherer JF, Edmond E, Simmonds P, Dickson JD, Bath GE. HTLV III antibody in Edinburgh drug addicts. Lancet 1985;ii:1129-30.

9 Urquhart GED, Scott SS, Wooldridge E. Human immunodeficiency virus (HIV) in intravenous drug abusers in Tayside. Commun Dis Scotland Wkly Rep 1987;CDS 87/09.

10 Taylor A, Frischer M, Green ST, et al. Low and stable prevalence of HIV among Glasgow drug injectors. Int $\mathcal{F}$ STD AIDS 1994;5:105-7.

11 Gore SM, Bird AG, Burns SM, et al. Drug injection and HIV prevalence in inmates of Glenochil prison. BMF 1995; 310:293-6.

12 Gore SM, Bird GA. Cross-sectional Willing Anonymous HIV Salivary (WASH) Surveillance studies and selfcompletion risk factor questionnaire in establishments of the Scottish Prison Service. ANSWER (AIDS News Supplement to the Weekly Report) AM-15 WR 95/39. 
13 Bird AG, Gore SM, Cameron S, Ross AJ, Goldberg D. Anonymous HIV surveillance with risk factor elicitation a Scotland's largest prison, Barlinnie. AIDS 1995;7:801-8.

14 Gore SM, Bird AG, Burns SM, Ross AJ, Goldberg DJ. Anonymous HIV and risk factor surveillance at Perth (for men) and Corntonvale (for women) prisons in Scotland. Int f STD AIDS 1997;8:166-75.

15 Goldberg D, Green ST, Taylor A, Frischer M, McKeganey $\mathrm{N}$. Comparison of four survey methods designed to estimate the prevalence of HIV among female prostitutes who inject drugs. Int $\mathcal{F}$ STD AIDS 1994;5:357-77.

16 Carr S, Goldberg DJ, Elliott L, et al. A primary health care service for Glasgow street sex workers - 6 years experience of
the 'drop-in centre'' 1989-1994. AIDS Care 1996;8:489-97.

17 Carr SV, Green ST, Goldberg D, et al. HIV prevalence among female street prostitutes attending a health care drop-in centre in Glasgow. AIDS 1992:6:1553-64.

18 Goldberg D, Davis B, Allardice G, McMenamin J. Monitoring the spread of HIV and AIDS in Scotland 1983-1994. Scott Med F 1996;41:131-8.

19 Goldberg D, Allardice G, McMenamin J, et al. Monitoring the spread of HIV and AIDS in Scotland 1983-1996.
ANSWER (AIDS News Supplement to the Weekly Report)

20 Taylor A, Goldberg D, Emslie J, et al. Outbreak of HIV infection in a Scottish prison. BMF 1995:310;289-92.

21 Frischer M, Elliott L, Taylor A, et al. Do needle exchanges help to control the spread of HIV among injecting drug users? AIDS 1993;7:1677-8.

22 Brettle RP. HIV and harm reduction for injection drug users. (Editorial review) AIDS 1991;5:125-36.

23 Gruer I, Cameron J, Elliott L. Building a city wide service for exchanging needles and syringes. BMF 1993;306:1394

24 Frischer $\mathrm{M}$, Bloor $\mathrm{M}$, Green $\mathrm{S}$, et al. Reduction in needle sharing among community wide samples of injecting drug sharing among community wide samples

25 Scott G, Goldberg D, Weir M, et al. HIV prevalence among heterosexuals attending genitourinary clinics in Scotlandheterosexuals attending genitourinary clinics

26 Unlinked Anonymous HIV Prevalence Monitoring Programme England and Wales. Report from the Unlinked Anonymous Surveys Steering Group. Department of Health. Data to the end of 1995 